\title{
Erratum: Kondo-Transport Spectroscopy of Single Molecule Magnets [Phys. Rev. Lett. 97, 206601 (2006)]
}

\author{
C. Romeike, M. R. Wegewijs, W. Hofstetter, and H. Schoeller \\ (Received 16 November 2010; published 7 January 2011)
}

DOI: 10.1103/PhysRevLett.106.019902

PACS numbers: 72.10.Fk, 75.10.Jm, 75.50.Xx, 75.60.Jk, 99.10.Cd

In Phys. Rev. Lett. 97, 206601 (2006), Ref. [1], we investigated the involvement of excited states in the Kondo effect in half-integer spin single-molecule magnets (SMM) in the limit of strong exchange interaction $J$. Unfortunately, the numerical results of this Letter are not correct. In particular, in the exchange interaction $J \mathbf{S} \cdot \mathbf{s}$, coupling the SMM to the first site on the Wilson chain in the NRG algorithm, the term $J S_{z} s_{z}$ was inadvertently implemented incorrectly. As a result we did not account for matrix elements of the $z$ component of the SMM spin operator $\left\langle i\left|S_{z}\right| j\right\rangle$ with $i \neq j$, while accounting for those with $i=j$. Here $|i\rangle,|j\rangle$ denote eigenstates of the SMM part of our Hamiltonian $\mathcal{H}_{\mathrm{SMM}}=-D S_{z}^{2}+$ $\frac{1}{2} B_{2}\left(S_{+}^{2}+S_{-}^{2}\right)+H_{z} S_{z}$ with $D>B_{2}$. Thus, the NRG algorithm was applied correctly but using incorrect matrix elements, thereby not capturing the transitions into SMM excited states. This only leads to incorrect results when two conditions are met: (i) the excited states of the SMM are relevant; i.e., $J$ is large enough compared to the largest anisotropy splitting $\Delta=(2 S-1) D$, and additionally (ii) there is transverse anisotropy, as in the Letter in question $\left(B_{2}\right)$, or a transverse magnetic field, as in [2]. Both conditions (i) and (ii) have to apply at the same time for quantitative errors to occur. Importantly, in the limit where $J$ is sufficiently weak compared to $\Delta$, where the Kondo effect in SMM was first studied [3], condition (i) does not apply and all results reported there are correct. This we checked by explicit recalculation of the results presented in [3] with the corrected code. Also, for large magnetic field $H_{z} \gg D, B_{2}$ the anisotropy becomes unimportant and the eigenstates approach spin eigenstates and the correct suppressed and split Kondo peaks are found. For the above reasons the problem could not be detected in the numerous checks we performed against known results for the Kondo effect for various spin $S$ in a magnetic field but without magnetic anisotropy.

Recalculation of the results of [1] confirm one of the central conclusions of that Letter, namely, that the excited states are involved in the Kondo effect. In Fig. 1 we show the dependence of the Kondo temperature on the transverse anisotropy $B_{2}$, correcting Fig. 2 in Ref. [1]. As the exchange interaction $J$ is increased $T_{K}$ grows. However, the correct order of magnitude of $T_{K}$ is much smaller than previously reported [1]. The discrepancy with the correct results in fact shows that for the parameters chosen the excited states are important even though the Kondo scale $T_{K}$ does not exceed the excitation energy $\Delta$. This implies that the two level picture which was valid in [3] does not apply for these parameters. Moreover, Fig. 1 shows no oscillatory dependence of $T_{K}$ on the magnetic anisotropy parameter $B_{2}$ as shown in Ref. [1]. We note that the cross check on Fig. 2(a) reported in Fig. 2(b) of Ref. [1] suffers from the same problem since the incorrect coupling matrix elements are used there as well (there was no problem with the NRG algorithm).

In Fig. 2 we show the dependence of the spectral function on the external longitudinal magnetic field $H_{z}$ (in units $g \mu_{B}=1$ ), correcting Fig. 3(b) in Ref. [1] for a $S=3 / 2$ SMM. The Kondo peak induced by the transverse anisotropy $B_{2}$ is rapidly suppressed by the field and splits by $2 S H_{z}$, i.e., $2 S$ times as fast as the usual Kondo $S=1 / 2$ peak without anisotropy. The reentrant behavior of the Kondo effect as function of a longitudinal magnetic field of Fig. 3(b) in Ref. [1] is

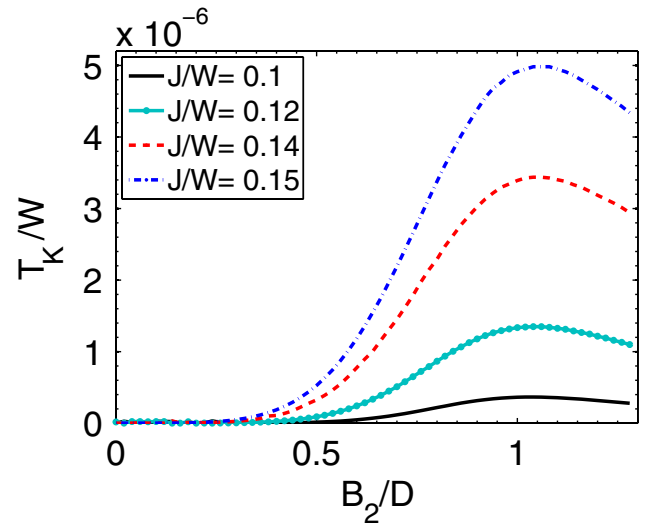

FIG. 1 (color online). Corrected figure 2(a) of the original Letter [1]. Dependence of the $T_{K}$ on the transverse anisotropy $B_{2}$ relative to $D$ for $S=7 / 2$ and $D=10^{-4} W$ where $W$ is the conduction bandwidth. 


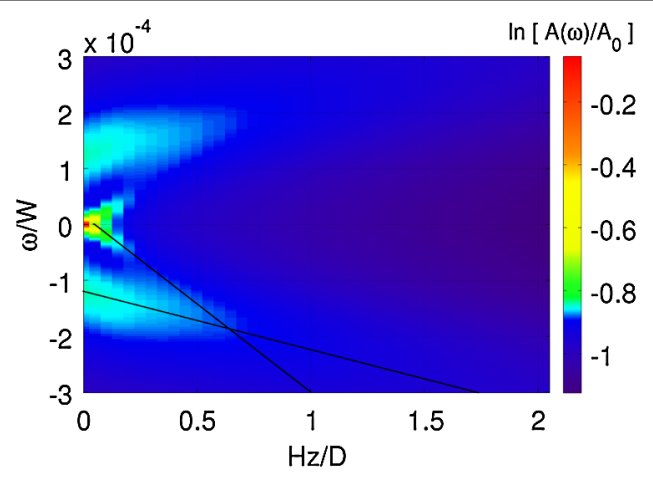

FIG. 2 (color online). Corrected figure 3(b) of the original Letter [1]. Logarithmic plot of the spectral function $A$ (normalization $A_{0}=\pi^{2} / 4$ ) as function of the frequency $\omega$ and the magnetic field energy $H_{z}$ for half-integer spin $S=3 / 2$ and $D=10^{-4} W$ and $B_{2} / D=0.1$. The value of $J=0.2$ is chosen somewhat larger than the original $J=0.15$ in order to better trace the field evolution of the weak Kondo peak which is indicated by the line starting from $\omega=0, H_{z}=0$ with slope $-2 S$. The line starting from the side peak at $\omega<0, H_{z}=0$ (zero-field splitting) indicates the evolution of the first excited state. In contrast to the original figure (plotted on much larger $\omega$ scale) the width of the Kondo peak is smaller than the zero-field splitting.

not present. The same conclusion holds for the Fig. 3(a),(c), and (d). We note that the zero-field split side peaks which evolve only with $H_{z}$ (instead of $2 \mathrm{SH}_{z}$ ) have recently been observed experimentally [4].

In view of the above a change of some of the main qualitative conclusions of Ref. [1] is warranted, which we now summarize: First, the dependence of the Kondo temperature on the transverse anisotropy $B_{2}$ is smooth, similar to the weak exchange limit studied correctly in Ref. [3], and shows no pronounced minima which correlate with the spin quantum number $S$ of the SMM. Secondly, there is no reentrant behavior of the Kondo effect as function of the longitudinal transverse field. Instead, such behavior occurs as function of a specific transverse field direction not studied in [1]. For these new results we refer to the erratum [5] to Ref. [2]. We mention that the time-dependent results of [6] are only quantitatively affected in limiting regimes [7]. Finally, for clarity we point out that the results on transport through SMMs of the authors other than those mentioned above are not affected in any way by the issue reported here.

All the new calculations above reported were performed by F. May when working at the Institut für Theoretische Physik, Johann Wolfgang Goethe-Universität, 60438 Frankfurt/Main, Germany.

[1] C. Romeike, M. R. Wegewijs, W. Hofstetter, and H. Schoeller, Phys. Rev. Lett. 97, 206601 (2006).

[2] M. R. Wegewijs, C. Romeike, H. Schoeller, and W. Hofstetter, New J. Phys. 9, 344 (2007).

[3] C. Romeike, M. R. Wegewijs, W. Hofstetter, and H. Schoeller, Phys. Rev. Lett. 96, 196601 (2006).

[4] A. S. Zyazin, J. W. van den Berg, E. A. Osorio, H. S. van der Zant, N. P. Konstantinidis, F. May, M. Leijnse, W. Hofstetter, M. R. Wegewijs, C. Danieli, and A. Cornia, Nano Lett. 10, 3307 (2010).

[5] M. R. Wegewijs, F. May, H. Schoeller, and W. Hofstetter, New J. Phys. (to be published).

[6] D. Roosen, M. R. Wegewijs, and W. Hofstetter, Phys. Rev. Lett. 100, 087201 (2008).

[7] D. Roosen, M. R. Wegewijs, and W. Hofstetter, Phys. Rev. Lett. 105, 259901 (2010). 